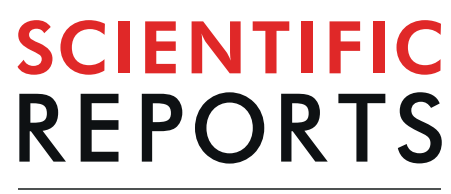

natureresearch

Check for updates

\title{
Re-evaluation of Streptococcus
} pneumoniae carriage in Portuguese elderly by qPCR increases carriage estimates and unveils an expanded pool of serotypes

\author{
Sónia T. Almeidaa ${ }^{1}$,ânia Pedro ${ }^{1}$, A. Cristina Paulo ${ }^{1}$, Hermínia de Lencastre ${ }^{2,3}$ \& \\ Raquel Sá-Leão ${ }^{1 凶}$
}

Streptococcus pneumoniae (pneumococcus) is a leading cause of infections worldwide. Disease is preceded by asymptomatic colonization of the upper respiratory tract. Classical culture-based methods (CCBM) suggest that colonization in the elderly is $<5 \%$. Recently, use of qPCR has challenged these observations. We estimated pneumococcal carriage prevalence and serotypes among Portuguese elderly using qPCR and compared results with those obtained by CCBM. Nasopharyngeal and oropharyngeal paired samples (599 each) of individuals over 60 years living in nursing $(n=299)$ or family $(n=300)$ homes were screened for the presence of pneumococci by qPCR targeting lyt $A$ and piaB. Positive samples were molecular serotyped. Use of qPCR improved detection of pneumococci in oropharyngeal samples compared to CCBM: from $0.7 \%$ to $10.4 \%(p<0.001)$ in the nursing home collection, and from $0.3 \%$ to $5.0 \%(p<0.001)$ in the family home collection. No significant differences were observed between both methods in nasopharyngeal samples $(5.4 \%$ vs. $5.4 \%$ in the nursing homes; and $4.3 \%$ vs. $4.7 \%$ in the family homes). Twenty-one serotypes/serogroups were detected by qPCR compared to 14 by CCBM. In conclusion, use of qPCR suggests that pneumococcal carriage in Portuguese elderly is approximately $10 \%$, and unveiled a large pool of serotypes. These results are important to understand progression to disease and impact of pneumococcal vaccines in the elderly.

Streptococcus pneumoniae (or pneumococcus) is a leading cause of infectious diseases worldwide, such as otitis media, pneumonia, bacteremia and meningitis. The incidence of pneumococcal infections is highest at the extremes of age affecting disproportionally young children and the elderly ${ }^{1-3}$. This latter group is increasingly important in our society as the world population is aging, resulting in an increasing demand for strategies to maintain quality-adjusted life years in advanced ages ${ }^{2}$.

To prevent pneumococcal disease three vaccines are currently available and several are under investigation ${ }^{4}$. These vaccines target 10,13 , or 23 serotypes of the over 95 described to date ${ }^{5}$. The 13 -valent pneumococcal conjugate vaccine (PCV13) is approved for all age groups and the 23 -valent pneumococcal polysaccharide vaccine (PPV23) is approved for individuals over 2 years of age. Several countries have issued recommendations for vaccination of adults over 65 years of age with one or both of these vaccines ${ }^{1,6,7}$.

In Portugal, PCVs for children were commercially available through the private market until mid-2015 (PCV7 became available in June 2001, PCV10 in April 2009, and PCV13 in January 2010). In August 2015, PCV13 was introduced in the National Immunization Plan for all children born after January $2015^{8}$. PPV23 and PCV13 are commercially available for adults but its usage has been low $(<10 \% \text { by } 2015)^{9,10}$.

Pneumococcal disease is always preceded by colonization of the upper respiratory tract, a phenomenon that is mostly asymptomatic ${ }^{11}$. Several studies have described high pneumococcal colonization rates (frequently over

${ }^{1}$ Laboratory of Molecular Microbiology of Human Pathogens, Instituto de Tecnologia Química e Biológica António Xavier, Universidade Nova de Lisboa, Oeiras, Portugal. ${ }^{2}$ Laboratory of Molecular Genetics, Instituto de Tecnologia Química e Biológica António Xavier, Universidade Nova de Lisboa, Oeiras, Portugal. ${ }^{3}$ Laboratory of Microbiology and

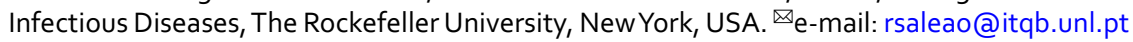


A

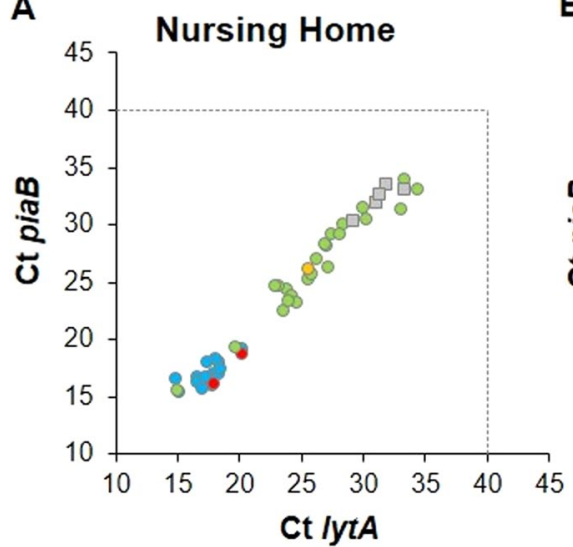

B

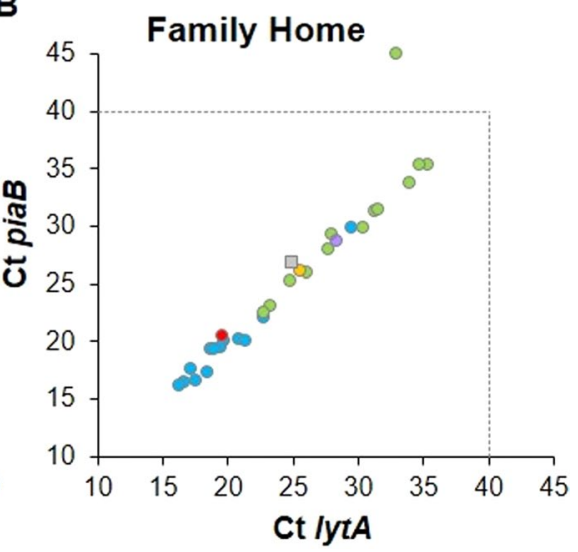

Figure 1. Detection of S. pneumoniae by real-time PCR in adults over 60 years of age living in nursing homes (A) and family homes (B). Blue circles, nasopharyngeal samples positive by culture; purple circles, nasopharyngeal samples negative by culture; red circles, oropharyngeal samples positive by culture; green circles, oropharyngeal samples negative by culture; yellow circles, positive control (S. pneumoniae TIGR4); grey squares, positive oropharyngeal samples in which the serotype was not determined. Dashed lines indicate the $\mathrm{Ct}$ value above which results were considered to be negative.

$60 \%)$ in young children ${ }^{12,13}$. By contrast, studies conducted in elderly populations have suggested, until recently, that pneumococcal colonization occurred at a very low prevalence $(1-5 \%)^{14-16}$. These studies relied on the use of classical culture-based approaches in which swabs of the nasopharynx and/or oropharynx were plated in selective media and pneumococcal presumptive colonies were sub-cultured for species identification ${ }^{17}$. This strategy, recommended by the WHO, has a good specificity but a low sensitivity ${ }^{17,18}$.

Recently, new strategies to detect pneumococcal carriage based on real-time PCR (qPCR) have been proposed, validated, and are now being increasingly used ${ }^{19-23}$. The use of qPCR enables high sensitivity in samples where pneumococci are at low density. With such approaches, carriage prevalence in the elderly has been re-evaluated with studies suggesting it may range between $0-20 \%$ in healthy individuals and reach approximately $50 \%$ period-prevalence (within 7-9 weeks) in individuals with influenza-like illness ${ }^{20-22,24}$.

In Portugal, the prevalence of pneumococcal carriage among the elderly has been studied by classical culture-based methods only. A previous study conducted by our group estimated a carriage prevalence of $2.3 \%{ }^{15}$.

The aim of this study was to re-evaluate the prevalence of nasopharyngeal and oropharyngeal colonization by S. pneumoniae in adults over 60 years of age using qPCR and compare the results with those obtained by classical culture-based approaches. We further characterized positive samples by serotyping and compared serotype distribution and diversity.

\section{Results}

Pneumococcal carriage. We evaluated pneumococcal carriage by real-time PCR targeting lyt $A$ and piaB genes in nasopharyngeal and oropharyngeal samples from 599 adults older than 60 years of age living in nursing homes $(n=299)$ and family homes $(n=300)$. Socio-demographic characteristics of the population were previously described ${ }^{15}$ and are summarized in Supplementary Table S1. In general, individuals living in a nursing home, were older, had less years of formal education, and were less active than those living in family homes. Results were compared to those obtained by classical culture-based methods.

$\mathrm{Ct}$ values for lyt $A$ and $p i a B$ genes were not significantly different between samples from adults living in nursing homes compared to those living in family homes (adjusted GLM, $\mathrm{p}=0.065$ for lyt $A ; \mathrm{p}=0.048$ for piaB). However, in both groups, the geometric Ct mean was significantly lower in positive nasopharyngeal samples than in positive oropharyngeal samples ( $\mathrm{p}<0.001$ for both $l y t A$ and $p i a B$ ), suggesting higher quantity of pneumococci in the harvests of cultures originating from nasopharyngeal samples compared to those originating from oropharyngeal samples (Fig. 1 and Supplementary Table S2).

The use of real-time PCR, when compared with classical culture-based methods, increased significantly the detection of pneumococcal carriage in oropharyngeal samples from $0.7 \%$ to $10.4 \%(\mathrm{p}<0.001)$ in the nursing home collection, and from $0.3 \%$ to $5.0 \%(\mathrm{p}<0.001)$ in the family home collection. By contrast, no significant differences were observed in carriage detection in nasopharyngeal samples when both methods were compared: $5.4 \%$ vs. $5.4 \%$ in the nursing home collection; and $4.3 \%$ vs. $4.7 \%(\mathrm{p}=1.0)$ in the family home collection (Table 1 and Fig. 2).

Overall, in adults living in nursing homes, $S$. pneumoniae was more frequently detected in oropharyngeal samples than in nasopharyngeal samples: $10.4 \%$ vs. $5.4 \%(p=0.0093)$, respectively; in adults living in family homes no significant differences were observed between the two sampling sites: $4.7 \%$ vs. $5.0 \%(\mathrm{p}=1.0)$ (Table 1$)$. When results of the two sampling sites were combined, the use of real-time PCR, compared to classical culture-based methods, increased significantly the detection of S. pneumoniae carriers from $5.7 \%$ to $12.7 \%(\mathrm{p}<0.001)$ in the nursing home collection and from $4.3 \%$ to $8.0 \%(\mathrm{p}=0.0026)$ in the family home collection (Table 1$)$.

Molecular serotyping. Molecular serotyping allowed assignment of a serotype/serogroup in 70 (40 from oropharynx and 30 from nasopharynx) of the $76 \mathrm{qPCR}$ positive samples. A single capsular type was detected in 


\begin{tabular}{|c|c|c|c|c|c|c|c|c|c|c|c|c|c|}
\hline \multirow[b]{3}{*}{ Collection } & \multirow[b]{3}{*}{$\begin{array}{l}\text { Participants } \\
\mathrm{n}\end{array}$} & \multicolumn{8}{|c|}{ Sampling Site n (\%) } & \multirow{2}{*}{\multicolumn{4}{|c|}{ Carriers n (\%) }} \\
\hline & & \multicolumn{4}{|c|}{ Oropharynx (OP) } & \multicolumn{4}{|c|}{ Nasopharynx (NP) } & & & & \\
\hline & & $\begin{array}{l}\text { Culture } \\
\text { positive }\end{array}$ & $\begin{array}{l}\text { qPCR } \\
\text { positive }\end{array}$ & $\begin{array}{l}\text { Culture } \\
\text { and/or } \\
\text { qPCR } \\
\text { positive }\end{array}$ & p-value & $\begin{array}{l}\text { Culture } \\
\text { positive }\end{array}$ & $\begin{array}{l}\text { qPCR } \\
\text { positive }\end{array}$ & $\begin{array}{l}\text { Culture } \\
\text { and/or } \\
\text { qPCR } \\
\text { positive }\end{array}$ & p-value & $\begin{array}{l}\text { Culture } \\
\text { positive } \\
\text { (NP and/ } \\
\text { or OP) }\end{array}$ & $\begin{array}{l}\text { qPCR } \\
\text { positive } \\
\text { (NP and/ } \\
\text { or OP) }\end{array}$ & Total & $\mathrm{p}$-value \\
\hline $\begin{array}{l}\text { Nursing } \\
\text { home }\end{array}$ & 299 & $\begin{array}{l}2 \\
(0.7 \%)\end{array}$ & $\begin{array}{l}31 \\
(10.4 \%)\end{array}$ & $\begin{array}{l}31 \\
(10.4 \%)\end{array}$ & $<0.001$ & $\begin{array}{l}16 \\
(5.4 \%)\end{array}$ & $\begin{array}{l}16 \\
(5.4 \%)\end{array}$ & $\begin{array}{l}16 \\
(5.4 \%)\end{array}$ & NA & $\begin{array}{l}17 \\
(5.7 \%)\end{array}$ & $\begin{array}{l}38 \\
(12.7 \%)\end{array}$ & $\begin{array}{l}38 \\
(12.7 \%)\end{array}$ & $<0.001$ \\
\hline $\begin{array}{l}\text { Family } \\
\text { home }\end{array}$ & 300 & $\begin{array}{l}1 \\
(0.3 \%)\end{array}$ & $\begin{array}{l}15 \\
(5.0 \%)\end{array}$ & $\begin{array}{l}15 \\
(5.0 \%)\end{array}$ & $<0.001$ & $\begin{array}{l}13 \\
(4.3 \%)\end{array}$ & $\begin{array}{l}14 \\
(4.7 \%)\end{array}$ & $\begin{array}{l}14 \\
(4.7 \%)\end{array}$ & 1.0 & $\begin{array}{l}13 \\
(4.3 \%)\end{array}$ & $\begin{array}{l}24 \\
(8.0 \%)\end{array}$ & $\begin{array}{l}24 \\
(8.0 \%)\end{array}$ & 0.003 \\
\hline
\end{tabular}

Table 1. Detection of S. pneumoniae carriers according to sampling site and methodology used. p-values determined using McNemar's Chi-squared test for paired individuals.

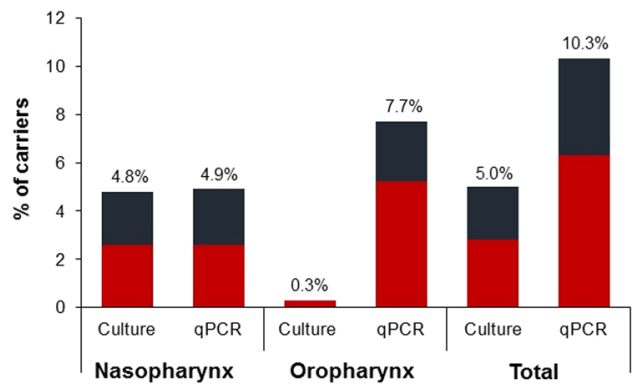

Figure 2. Proportion of pneumococcal carriers detected by classical culture-based methods or real-time PCR. Red bars, adults over 60 years of age living in nursing homes; blue bars, adults over 60 years of age living in family homes.

67 qPCR pneumococcal positive samples and two serotypes were detected in three samples: one individual was co-colonized with serotypes $19 \mathrm{~A}$ and $22 \mathrm{~A} / \mathrm{F}$ which were detected both in the nasopharynx and the oropharynx; other individual was co-colonized with serotypes 3 and $23 \mathrm{~A}$ in the oropharynx. The six samples for which a serotype could not be assigned were all from the oropharynx (Fig. 1). Failure of assigning a capsular type to these samples suggests that they may have not been targeted by the primer set tested.

In total, twenty-one different serotypes/serogroups were detected among 76 samples that were positive for pneumococci by qPCR compared to 14 serotypes present in the 32 samples that were positive for pneumococci by classical culture-based methods. Serotype diversity (GSID $=0.897,95 \%$ CI: 0.896-0.898) was slightly higher among samples detected by qPCR compared to those by classical culture-based methods (GSID $=0.879,95 \% \mathrm{CI}$ : 0.877-0.881). Serotypes/serogroups $15 \mathrm{~A} / \mathrm{F}, 24$ and 38, which are not included in any pneumococcal multi-valent vaccine, and serotypes/serogroups 7A/F, 10A, 12A/B/F and 20 potentially targeted by PPV23 (7F is also targeted by PCV13), were only detected in qPCR positive samples (Table 2).

Combined nasopharyngeal and oropharyngeal sampling did not yield significantly more different serotypes than each sampling site alone (14 serotypes/serogroups detected in nasopharyngeal samples and 17 detected in the oropharyngeal samples, $\mathrm{p}=0.166$ and $\mathrm{p}=0.564$, respectively).

The most frequent serotypes/serogroups were 19A $(n=17), 23 \mathrm{~A}(\mathrm{n}=9), 35 \mathrm{~F}(\mathrm{n}=7), 6 \mathrm{C} / \mathrm{D}(\mathrm{n}=6), 22 \mathrm{~A} / \mathrm{F}$ $(\mathrm{n}=5)$ and $11 \mathrm{~A} / \mathrm{D}(\mathrm{n}=4)$, which, together, accounted for $68.6 \%$ of all serotyped samples (Table 2). Serotypes $7 B / C(n=1), 9 L(n=1), 23 B(n=2)$ and $31(n=1)$ were only found in nasopharyngeal samples, while serotypes $7 \mathrm{~A} / \mathrm{F}(\mathrm{n}=1), 10 \mathrm{~A}(\mathrm{n}=1), 12 \mathrm{~A} / \mathrm{B} / \mathrm{F}(\mathrm{n}=1), 15 \mathrm{~A} / \mathrm{F}(\mathrm{n}=1), 20(\mathrm{n}=2), 24(\mathrm{n}=3)$ and $38(\mathrm{n}=1)$ were only found in oropharyngeal samples (Table 2 ).

Overall, among the 62 carriers (38 living in nursing homes and 24 living in family homes) a maximum of $25.8 \%$ and $46.8 \%$ had serotypes potentially covered by PCV13, and PPV23, respectively.

Of note, within nursing homes, there was evidence of cross-transmission with a common serotype being frequently isolated from multiple individuals (Fig. 3). This contributed to a lower serotype diversity in the nursing home collection (GSID $=0.778,95 \% \mathrm{CI}$ : $0.775-0.782$ ) when compared to the family home collection $(\mathrm{GSID}=0.919,95 \%$ CI: $0.918-0.920)$.

\section{Discussion}

In this study qPCR targeting lytA and piaB was used to investigate the prevalence of nasopharyngeal and oropharyngeal pneumococcal carriage in two collections of samples obtained from adults over 60 years of age living in either nursing homes or family homes. Positive samples were further characterized by molecular serotyping. These collections were previously characterized by classical culture-based methods as part of a much larger pneumococcal carriage study ${ }^{15}$.

In our previous study, as well as in studies from countries such as Belgium, Finland and Israel - which also used conventional culture followed by identification of presumptive colonies S. pneumoniae - pneumococcal carriage rates were estimated to be in the range of 3-5\% among the elderly ${ }^{14-16,25}$. 


\begin{tabular}{|c|c|c|c|c|c|}
\hline \multirow[b]{3}{*}{$\begin{array}{l}\text { Serotype/ } \\
\text { serogroup }\end{array}$} & \multirow[b]{3}{*}{ Vaccine $\mathrm{e}^{\mathrm{a}}$} & \multicolumn{4}{|c|}{ Number of samples with serotype/serogroup } \\
\hline & & \multicolumn{2}{|c|}{ Nursing homes } & \multicolumn{2}{|l|}{ Family home } \\
\hline & & $\begin{array}{l}\text { Classical culture- } \\
\text { based approaches }\end{array}$ & $\mathbf{q P C R}^{\mathrm{c}}$ & $\begin{array}{l}\text { Classical culture- } \\
\text { based approaches }\end{array}$ & qPCR $^{\mathrm{C}}$ \\
\hline 3 & PCV13/PPV23 & - & - & 2 & 3 \\
\hline 5 & PCV13/PPV23 & - & $1^{\mathrm{d}}$ & - & - \\
\hline $6 \mathrm{~A}$ & PCV13/PPV23 & - & - & 2 & 2 \\
\hline $6 \mathrm{C} / \mathrm{D}$ & - & - & 2 & $2(6 \mathrm{C})$ & 4 \\
\hline $7 \mathrm{~A} / \mathrm{F}$ & PCV13/PPV23 & - & - & - & 1 \\
\hline $7 \mathrm{~B} / \mathrm{C}$ & - & - & - & 1 & 1 \\
\hline 9L & - & - & - & 1 & 1 \\
\hline $10 \mathrm{~A}$ & PPV23 & - & - & - & 1 \\
\hline $11 \mathrm{~A} / \mathrm{D}$ & PPV23 & - & 3 & $1(11 \mathrm{~A})$ & 1 \\
\hline $12 \mathrm{~A} / \mathrm{B} / \mathrm{F}$ & PPV23 & - & - & - & 1 \\
\hline $15 \mathrm{~A} / \mathrm{F}$ & - & - & - & - & 1 \\
\hline $16 \mathrm{~F}$ & - & - & - & 1 & 2 \\
\hline $17 \mathrm{~F}$ & PPV23 & 1 & 3 & - & - \\
\hline 19A & PCV13/PPV23 & 7 & 16 & - & 1 \\
\hline 20 & PPV23 & - & 2 & - & - \\
\hline $22 \mathrm{~A} / \mathrm{F}$ & PPV23 & $2(22 \mathrm{~F})$ & 4 & - & 1 \\
\hline $23 \mathrm{~A}$ & - & 4 & 6 & 2 & 3 \\
\hline $23 \mathrm{~B}$ & - & 1 & 1 & 1 & 1 \\
\hline 24 & - & - & 1 & - & 2 \\
\hline 31 & - & - & - & 1 & 1 \\
\hline $35 \mathrm{~F}$ & - & 3 & 6 & 1 & 1 \\
\hline 38 & - & - & - & - & 1 \\
\hline ND & - & - & 5 & - & 1 \\
\hline
\end{tabular}

Table 2. S. pneumoniae serotypes/serogroups detected in samples obtained from adults over 60 years old.

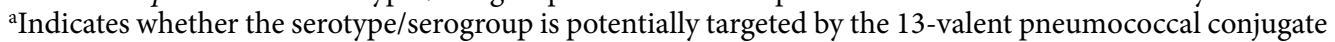
vaccine (PCV13: 1, 3, 4, 5, 6A, 6B, 7F, 9V, 14, 18C, 19A, 19F and 23F) or by the 23-valent polysaccharide vaccine (PPV23: 1, 2, 3, 4, 5, 6B, 7F, 8, 9N, 9V, 10A, 11A, 12F, 14, 15B, 17F, 18C, 19A, 19F, 20, 22F, 23F and 33F).

${ }^{b}$ Detection of pneumococci by identification and characterization of presumptive colonies grown on gentamicin blood agar, as described in the Methods section. ${ }^{c}$ Detection of pneumococci using a qPCR targeting lyt $A$ and piaB genes, as described in the Methods section. ${ }^{\mathrm{d}}$ Given that this specific assay is known to yield false positive results, it was not taken into consideration. ND, not determined.

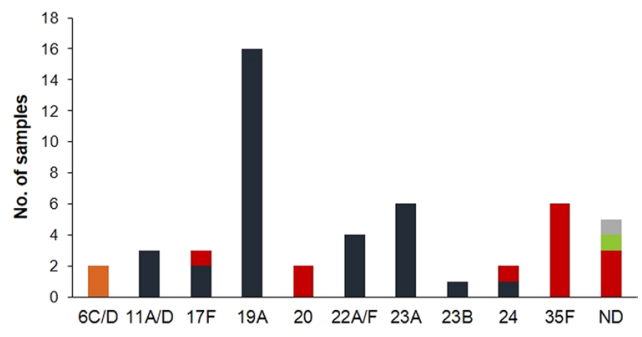

Figure 3. Serotype distribution of pneumococcal isolates carried by adults over 60 years of age living in nursing homes. Different colors indicate different nursing homes.

In this study, use of qPCR increased the detection of pneumococci in oropharyngeal samples by approximately 15 times in both the nursing home and family home collections. By contrast, there was no significant added value in using qPCR to detect pneumococci in nasopharyngeal samples. The use of qPCR is known to be particularly valuable to detect pneumococci in samples that are highly polymicrobial and/or in which pneumococci are present at low density. In oropharyngeal samples both conditions tend to be common ${ }^{19,20,22}$. The nasopharynx, by contrast, is known to be less polymicrobial and pneumococci, when present, tend to thrive $\mathrm{e}^{15,26-29}$.

When all results were compared, pneumococci were more frequently detected in oropharyngeal samples than in nasopharyngeal samples $(7.7 \%$ vs. $5.0 \%$, McNemar's, $\mathrm{p}<0.05)$ and there were also twice more individuals positive for pneumococci exclusively in oropharyngeal samples $(n=32)$ compared to individuals positive exclusively in nasopharyngeal samples $(n=16)$. These observations suggest that pneumococci, in the elderly, tend to preferentially colonize the oropharynx. 
They also support the recommendation that to detect pneumococci in senior adults it is important to combine both nasopharyngeal and oropharyngeal samples and that qPCR (targeting more than one validated gene, such as $l y t A$ and piaB) should be used when analyzing oropharyngeal samples ${ }^{19,20,22}$. In our study, half of the pneumococcal carriers were only detected in these conditions.

Overall, we estimated that approximately $10 \%$ of the individuals were pneumococcal carriers independently of whether they lived in a nursing home or in a family home. These estimates are lower than those described in studies conducted in Italy or the Netherlands, which were closer to $20 \%{ }^{22,24}$. These differences are likely to be due to seasonality: the latter studies were carried out in winter or winter/spring while ours took place throughout the year. In fact, a variation between $0-17 \%$ in pneumococcal carriage prevalence among adults over 65 years of age was noted in the US longitudinal study of Branche et al. ${ }^{21}$.

While we did not observe differences in density of colonization between individuals living in nursing homes or family homes, we observed (unsurprisingly) lower serotype diversity and evidence for cross-transmission within nursing homes. These are settings where individuals are often confined and where transmission of infectious agents is often facilitated ${ }^{30}$.

Molecular serotyping of qPCR pneumococcal positive samples enabled assignment of a serotype/serogroup to most (92\%) samples. Furthermore, the number of different serotypes that were detected increased by $50 \%$ ( 21 in qPCR samples vs. 14 by classical culture-based processed samples) expanding considerably the pool of serotypes detected in the population. This included non-vaccine serotypes and vaccine serotypes that, otherwise, would remain undetected and that may be important to monitor intervention strategies such as the use of pneumococcal vaccines.

In conclusion, carriage of pneumococci in senior adults is significantly higher than the one estimated by classical culture-based methods alone. Our current estimates suggest that, in Portugal, it is approximately $10 \%$. The high number of serotypes circulating in this population and the transmission observed within nursing homes warrant additional studies aimed to assess its implications in disease. As the number and proportion of aged individuals increases in societies worldwide, accurate estimates of pneumococcal carriage prevalence and circulating serotypes in this group are crucial to better understand colonization dynamics, serotype disease potential, and impact of pneumococcal vaccines in this age group.

\section{Methods}

Study participants and samples. This study was nested on a study previously described ${ }^{15}$. Briefly, pneumococcal carriage prevalence and associated risk factors were studied among 3,361 adults older than 60 years of age, between April 2010 and December of 2012. For each participant, one nasopharyngeal and one oropharyngeal sample were obtained. Pneumococci were isolated and identified by routine procedures based on culture-based methods ${ }^{31,32}$, and serotyped by conventional multiplex PCR and/or by the Quellung reaction ${ }^{33,34}$. Briefly, in the initial study, swabs were plated on gentamycin blood agar and incubated overnight at $37^{\circ} \mathrm{C}$ in anaerobiose jars. On the following day, suspected pneumococcal colonies were isolated and identified by routine culture methods. All the remaining total bacterial growth was collected and frozen at $-80^{\circ} \mathrm{C}$.

In the current study, two subsets of samples of the initial study were re-examined. One set corresponded to 600 paired samples (300 nasopharyngeal and 300 oropharyngeal obtained from 300 individuals) randomly selected from a pool previously obtained from 3,062 individuals living in family homes. The second set corresponded to 598 samples (299 nasopharyngeal and 299 oropharyngeal) obtained from all individuals living in nursing homes at the time of the study ${ }^{15}$.

Identification and serotyping of S. pneumoniae by molecular methods. Total bacterial growth was thawed on ice, vortexed for 20 seconds and $200 \mu \mathrm{l}$ were transferred into a sample tube with $200 \mu$ of a lysis buffer (MagNA Pure Compact Nucleic Acid Isolation Kit, Roche Diagnostics GmbH), and incubated for 20 min at $37^{\circ} \mathrm{C}$. Total DNA was then extracted using the MagNA Pure Compact instrument (Roche Diagnostics $\mathrm{GmbH}$ ) as recommended by the manufacturer.

Pneumococcal carriage was evaluated by real-time PCR, targeting two pneumococcal genes: lytA (major pneumococcal autolysin) and piaB (iron uptake $\mathrm{ABC}$ transporter lipoprotein $\mathrm{PiaB})^{19,35}$. Reactions for both genes were performed in a final volume of $25 \mu \mathrm{l}$, containing 1x FastStart TaqMan Probe Master (Roche), $150 \mathrm{nM}$ of each primer, $75 \mathrm{nM}$ of probe and $2.5 \mu \mathrm{l}$ of total DNA. DNA amplification was performed in the CFX96 Real-Time System Amplification (Bio-Rad) using the following conditions: $95^{\circ} \mathrm{C}$ for $10 \mathrm{~min}$, followed by 45 cycles of $95^{\circ} \mathrm{C}$ for $15 \mathrm{sec}$ and $60^{\circ} \mathrm{C}$ for $1 \mathrm{~min}$. Samples were considered positive for pneumococci when both genes had cycle threshold $(\mathrm{Ct})$ values below 40 .

S. pneumoniae capsular types were accessed in positive samples by uniplex qPCR following a pooling strategy as previously described ${ }^{36}$. Pools of five samples containing $2 \mu$ of each sample were tested. Each pool was used as template for qPCR in a total volume of $25 \mu \mathrm{l}$. If a $\mathrm{Ct}<40$ was obtained in a given pool of samples, these were tested individually for the serotype that originated the signal. In this latter case, $2.5 \mu \mathrm{l}$ of DNA was used per reaction. Samples were considered positive for a given serotype when Ct values were below 40. A panel of 23 sets of primers and probes was used targeting serotypes or serogroups: $1,2,3,4,5,6 \mathrm{~A} / 6 \mathrm{~B} / 6 \mathrm{C} / 6 \mathrm{D}, 7 \mathrm{~A} / \mathrm{F}, 9 \mathrm{~A} / \mathrm{V}, 11 \mathrm{~A} / \mathrm{D}$, $12 \mathrm{~A} / 12 \mathrm{~B} / 12 \mathrm{~F} / 44 / 46,14,15 \mathrm{~A} / \mathrm{F}, 16 \mathrm{~F}, 18 \mathrm{~A} / 18 \mathrm{~B} / 18 \mathrm{C} / 18 \mathrm{~F}, 19 \mathrm{~A}, 19 \mathrm{~F}, 22 \mathrm{~A} / \mathrm{F}, 23 \mathrm{~A}, 23 \mathrm{~F}, 33 \mathrm{~A} / 33 \mathrm{~F} / 37^{37}, 8,10 \mathrm{~A} / \mathrm{B}$ and $38^{38}$. Samples were considered positive for a given serotype when $\mathrm{Ct}$ values were below 40 . Following previous studies that indicated that non-pneumococcal streptococci in the oropharynx can confound molecular serotyping assays, we disregarded positive results obtained by qPCR for serotypes 4 and $5^{20,26,39}$. In addition, conventional multiplex PCR was used to detect serotypes 7B/7C/40, 9N/L, 15B/C, 17F, 20, 21, 23B, 24, 31, 34, 35B, 35F/47F and non-typeables using primers previously described ${ }^{33,40}$. 
Statistical analysis. The geometric mean was used to summarize the distribution of Ct values from lytA and piaB of nasopharyngeal and oropharyngeal samples. To look for associations between Ct values from nursing home $v$ f family home and nasopharynx vs oropharynx an adjusted generalized linear model (GLM) using a Gaussian distribution and a log link function was used.

The McNemar's test was used to compare culture and real-time PCR methods based on paired individuals. The Chi-square test was used to compare prevalence of $S$. pneumoniae between nasopharyngeal and oropharyngeal samples and between nursing home and family home by real-time PCR or culture-based methods. A p-value of $<0.05$ was considered statistically significant for all the tests used. The Gini-Simpson index of diversity (GSID) was used to calculate serotype diversity. All analyses were performed using R version 3.2.3 ${ }^{41}$.

Ethics statement. This study was conducted in accordance with the European Statements for Good Clinical Practice and the declaration of Helsinki of the World Health Medical Association. In addition, it was registered and approved at health care centers of Oeiras and Montemor-o-Novo that report to Administração Regional de Saúde (ARS, "Regional Health Administration") of Lisboa e Vale do Tejo, and Alentejo, respectively, from the Ministry of Health. Informed written consent was obtained from all participants. All samples and questionnaires were attributed a numeric code and were processed anonymously.

Received: 21 February 2020; Accepted: 27 April 2020;

Published online: 20 May 2020

\section{References}

1. CDC. Prevention of pneumococcal disease: recommendations of the advisory committee on the immunization practices (ACIP). Morbidity and Mortality Weekly Report (MMWR) 46, 1-24 (1997).

2. Ludwig, E., Bonanni, P., Rohde, G., Sayiner, A. \& Torres, A. The remaining challenges of pneumococcal disease in adults. Eur Respir Rev 21, 57-65 (2012).

3. WHO. The top 10 causes of death. Geneva: World Health Organization, http://www.who.int/mediacentre/factsheets/fs310/en/ index2.html [updated in June 2016]. Accessed on 21 July, 2019] (2013).

4. Converso, T. R., Assoni, L., André, G. O., Darrieux, M. \& Leite, L. C. C. The long search for a serotype independent pneumococcal vaccine. Expert Rev Vaccines, https://doi.org/10.1080/14760584.2020.1711055 (2020).

5. Park, I. H. et al. Genetic, biochemical, and serological characterization of a new pneumococcal serotype, $6 \mathrm{H}$, and generation of a pneumococcal strain producing three different capsular repeat units. Clin Vaccine Immunol 22, 313-318, https://doi.org/10.1128/ CVI.00647-14 (2015).

6. Licensure, CDC. of 13-valent pneumococcal conjugate vaccine for adults aged 50 years and older. MMWR Morb Mortal Wkly Rep 61, 394-395 (2012).

7. Direcção Geral de Saúde. Norma 11/2015-Vacinação contra infecções por Streptococcus pneumoniae de grupos com risco acrescido para doença invasiva pneumocócica (DIP). Adultos ( $\geq 18$ anos de idade). (2015).

8. Direcção Geral de Saúde. Norma 08/2015-Programa Nacional de Vacinação. Introdução da vacina conjugada de 13 valências contra infeções por Streptococcus pneumoniae (Pn13). (2015).

9. Horácio, A. N., Diamantino-Miranda, J., Aguiar, S. I., Ramirez, M. \& Melo-Cristino, J. Serotype changes in adult invasive pneumococcal infections in Portugal did not reduce the high fraction of potentially vaccine preventable infections. Vaccine 30, 218-224 (2012)

10. Kislaya, I. et al. Indirect effect of 7-valent and 13-valent pneumococcal conjugated vaccines on pneumococcal pneumonia hospitalizations in elderly. PLoS One 14, e0209428 (2019).

11. Simell, B. et al. The fundamental link between pneumococcal carriage and disease. Expert Rev Vaccines 11, 841-855 (2012).

12. Bosch, A. A. T. M. et al. Nasopharyngeal carriage of Streptococcus pneumoniae and other bacteria in the $7^{\text {th }}$ year after implementation of the pneumococcal conjugate vaccine in the Netherlands. Vaccine 34, 531-539 (2016).

13. Nunes, S. et al. The impact of private use of PCV7 in 2009 and 2010 on serotypes and antimicrobial resistance of Streptococcus pneumoniae carried by young children in Portugal: Comparison with data obtained since 1996 generating a 15-year study prior to PCV13 introduction. Vaccine 34, 1648-1656 (2016).

14. Flamaing, J., Peetermans, W. E., Vandeven, J. \& Verhaegen, J. Pneumococcal colonization in older persons in a nonoutbreak setting. J Am Geriatr Soc 58, 396-398 (2010).

15. Almeida, S. T. et al. Low prevalence of pneumococcal carriage and high serotype and genotype diversity among adults over 60 years of age living in Portugal. PLoS One 9, e90974 (2014).

16. Palmu, A. A., Kaijalainen, T., Saukkoriipi, A., Leinonen, M. \& Kilpi, T. M. Nasopharyngeal carriage of Streptococcus pneumoniae and pneumococcal urine antigen test in healthy elderly subjects. Scand J Infect Dis 44, 433-438 (2012).

17. Satzke, C. et al. Standard method for detecting upper respiratory carriage of Streptococcus pneumoniae: updated recommendations from the World Health Organization Pneumococcal Carriage Working Group. Vaccine 32, 165-179 (2013).

18. Chien, Y. W. et al. Density interactions among Streptococcus pneumoniae, Haemophilus influenzae and Staphylococcus aureus in the nasopharynx of young Peruvian children. Pediatr Infect Dis J 32, 72-77 (2013).

19. Trzciński, K. et al. Superiority of trans-oral over trans-nasal sampling in detecting Streptococcus pneumoniae colonization in adults. PLoS One 8, e60520 (2013).

20. Krone, C. L. et al. Carriage of Streptococcus pneumoniae in aged adults with influenza-like-illness. PLoS One 10, e0119875 (2015).

21. Branche, A. R. et al. Effect of prior vaccination on carriage rates of Streptococcus pneumoniae in older adults: A longitudinal surveillance study. Vaccine 36, 4304-4310 (2018).

22. van Deursen, A. M., van den Bergh, M. R., Sanders, E. A. \& Group, C. P. S. Carriage of Streptococcus pneumoniae in asymptomatic, community-dwelling elderly in the Netherlands. Vaccine 34, 4-6 (2016).

23. Tavares, D. A. et al. Identification of Streptococcus pneumoniae by a real-time PCR assay targeting SP2020. Sci Rep 9, 3285 (2019).

24. Ansaldi, F. et al. Carriage of Streptoccoccus pneumoniae in healthy adults aged $60 \mathrm{y}$ or over in a population with very high and longlasting pneumococcal conjugate vaccine coverage in children. Rationale and perspectives for PCV13 implementation. Human Vaccines \& Immunotherapeutics 9, 614-620 (2013).

25. Regev-Yochay, G. et al. Nasopharyngeal carriage of Streptococcus pneumoniae by adults and children in community and family settings. Clin Infect Dis 38, 632-639 (2004).

26. Carvalho Mda, G. et al. Non-pneumococcal mitis-group streptococci confound detection of pneumococcal capsular serotypespecific loci in upper respiratory tract. PeerJ 1, e97 (2013).

27. Watt, J. P. et al. Nasopharyngeal versus oropharyngeal sampling for detection of pneumococcal carriage in adults. J Clin Microbiol 42, 4974-4976 (2004).

28. Man, W. H., de Steenhuijsen Piters, W. A. \& Bogaert, D. The microbiota of the respiratory tract: gatekeeper to respiratory health. Nat Rev Microbiol 15, 259-270 (2017). 
29. Charlson, E. S. et al. Disordered microbial communities in the upper respiratory tract of cigarette smokers. PLoS One 5, e15216 (2010).

30. Nuorti, J. P. et al. An outbreak of multidrug-resistant pneumococcal pneumonia and bacteremia among unvaccinated nursing home residents. N Engl J Med 338, 1861-1868 (1998).

31. Nunes, S. et al. Trends in drug resistance, serotypes, and molecular types of Streptococcus pneumoniae colonizing preschool-age children attending day care centers in Lisbon, Portugal: a summary of 4 years of annual surveillance. J Clin Microbiol 43, 1285-1293 (2005).

32. Sá-Leão, R. et al. Genetic diversity and clonal patterns among antibiotic-susceptible and -resistant Streptococcus pneumoniae colonizing children: day care centers as autonomous epidemiological units. J. Clin. Microbiol 38, 4137-4144 (2000).

33. Pai, R., Gertz, R. E. \& Beall, B. Sequential multiplex PCR approach for determining capsular serotypes of Streptococcus pneumoniae isolates. J. Clin. Microbiol. 44, 124-131 (2006).

34. Sorensen, U. B. Typing of pneumococci by using 12 pooled antisera. J Clin Microbiol 31, 2097-2100 (1993).

35. Carvalho Mda, G. et al. Evaluation and improvement of real-time PCR assays targeting lytA, ply, and psaA genes for detection of pneumococcal DNA. J Clin Microbiol 45, 2460-2466 (2007).

36. Wyllie, A. L. et al. Molecular surveillance on Streptococcus pneumoniae carriage in non-elderly adults; little evidence for pneumococcal circulation independent from the reservoir in children. Sci Rep 6, 34888 (2016)

37. Pimenta, F. C. et al. Sequential triplex real-time PCR assay for detecting 21 pneumococcal capsular serotypes that account for a high global disease burden. J Clin Microbiol 51, 647-652 (2013).

38. Azzari, C. et al. Realtime PCR is more sensitive than multiplex PCR for diagnosis and serotyping in children with culture negative pneumococcal invasive disease. PLoS One 5, e9282 (2010).

39. Wyllie, A. L. et al. Streptococcus pneumoniae in saliva of Dutch primary school children. PLoS One 9, e102045 (2014).

40. CDC. Conventional PCR Serotype Deduction Protocols, http://www.cdc.gov/streplab/pcr.html [Accessed 6 January, 2020] (2010).

41. R Core Team. R: A language and environment for statistical computing. (R Foundation for Statistical Computing. Available online at, https://www.R-project.org/. 2015).

\section{Acknowledgements}

This work was partially supported through Projects LISBOA-01-0145-FEDER-007660 (Microbiologia Molecular, Estrutural e Celular, funded by FEDER funds through COMPETE2020 - Programa Operacional Competitividade e Internacionalização (POCI) and LISBOA-01-0145-FEDER-016417 (ONEIDA project, co-funded by FEEI "Fundos Europeus Estruturais e de Investimento" from "Programa Operacional Regional Lisboa 2020"). STA and ACP were supported by grants SFRH/BD/108380/2015 and SFRH/PBD/99638/2014 from Fundação para a Ciência e a Tecnologia, Portugal. The authors thank all the participants that collaborated in the study. We thank nurses Anabela Gonçalves and Pedro Fonseca for collecting the nasopharyngeal and oropharyngeal samples. We are grateful to Ana Paula Ribeiro for her support and enthusiasm during fieldwork carried out in Montemor-oNovo.

\section{Author contributions}

The study was conceived by R.S.L. Experiments were done by S.T.A. and T.P. and statistical analyses was done by A.C.P. Data interpretation was done by S.T.A., A.C.P. and R.S.L. R.S.L. and H.d.L. contributed with reagents and materials. The manuscript was written by S.T.A. and R.S.L. and critically revised by all authors. All authors read and approved the final version of the manuscript.

\section{Competing interests}

R.S.L. has received consulting and speaking fees from Pfizer and consulting fees from Merck Sharp \& Dome. R.S.L. has received funds for unrestricted research grants from Pfizer, paid directly to her institution. The other authors declare no conflict of interest.

\section{Additional information}

Supplementary information is available for this paper at https://doi.org/10.1038/s41598-020-65399-x.

Correspondence and requests for materials should be addressed to R.S.-L.

Reprints and permissions information is available at www.nature.com/reprints.

Publisher's note Springer Nature remains neutral with regard to jurisdictional claims in published maps and institutional affiliations.

(c) (i) Open Access This article is licensed under a Creative Commons Attribution 4.0 International License, which permits use, sharing, adaptation, distribution and reproduction in any medium or format, as long as you give appropriate credit to the original author(s) and the source, provide a link to the Creative Commons license, and indicate if changes were made. The images or other third party material in this article are included in the article's Creative Commons license, unless indicated otherwise in a credit line to the material. If material is not included in the article's Creative Commons license and your intended use is not permitted by statutory regulation or exceeds the permitted use, you will need to obtain permission directly from the copyright holder. To view a copy of this license, visit http://creativecommons.org/licenses/by/4.0/.

(C) The Author(s) 2020 